\title{
A rapid, convient and diversified-multicomponent synthesis and characterization of pyrano[2,3-b]indoles
}

\author{
H. B. Ghodasara*, P. M. Singala, V. H. Shah** \\ Department of Chemistry, Saurashtra University, University Road, Rajkot 360 005, Gujarat, India \\ *,**E-mail address: hbchem1989@gmail.com , shah_v_h@yahoo.com
}

\begin{abstract}
A series of pyrano[2,3-b]indoles, was efficiently synthesized via one-pot, multi component reaction (MCRs) of 1,3-bifunctional synthon(malononitrile/ethylcyano acetate), aromatic aldehydes and oxiindole in the presence of various basic catalyst. The key advantages of this process are high yields, shorter reaction times, easy work-up, and purification of products by non-chromatographic method.
\end{abstract}

Keywords: 1,3-bifunctional synthon; Pyrano[2,3-b]indoles; multi component reaction; nonchromatographic method

\section{INTRODUCTION}

Multi component reactions (MCRs) have more importance, great interest enjoying an outstanding status in modern organic synthesis and medicinal chemistry because they are one-pot processes bringing together three or more components and show high atom economy and high selectivity ${ }^{1,2}$. MCRs comply with the principles of green chemistry in terms of economy of steps as well as many of the rigid criteria of an ideal organic synthesis. These reactions are effective in construction highly functionalized small organic molecules from readily available starting materials in a single step with natural flexibility for creating molecular complexity and diversity coupled with minimization of time, labor, cost and waste production $^{3-6}$. The pyran nucleus is a fertile source of biologically important molecules. Compounds containing this moiety have many pharmacological properties and play important roles in biochemical processes. They are reported to possess a multiplicity of pharmacological properties including anticancer ${ }^{7}$, antimicrobial ${ }^{8}$, anti-inflammatory ${ }^{9}$, insecticidal, and molluscicidal activities ${ }^{10,11}$. They are also potential inhibitors of human Chk1 kinase ${ }^{12}$. They also find applications as pharmaceutical ingredients and biodegradable agrochemicals $^{13}$.

In a view of great importance of pyran derivatives, various methods for synthesis of fused pyran derivatives have been reported. During the last few years, some methods were introduced for the synthesis of these compounds. Pyran were first obtained in 1973 by reaction between 3-methyl-1 phenylpyrazolin-5-one and tetracyanoethylene ${ }^{14}$. After this Otto had proposed the synthesis of the dihydropyrano[2,3-c]pyrazoles in 1974, via the base 
catalyzed cycloaddition of 4-aryliden-5-pyrazolone ${ }^{15}$. Sharanin and research group have developed a three-component reaction between pyrazolone, an aldehyde and malononitrile in ethanol using triethylamine as the catalyst ${ }^{16}$. Kappusami and co-workers have developed solvent-free multicomponent synthesis of pyranopyrazoles using per-6-amino- $\beta$-cyclodextrin as a catalyst ${ }^{17}$. More recently Myrboh et al. reported the synthesis of pyranopyrazoles using L-proline and $\gamma$-alumina as catalyst ${ }^{18}$. As a part of ongoing program on the development of novel methods in organic synthesis, we report herein a simple, rapid and high yielding one pot three component reaction protocol for the synthesis of pyrano[2,3-b]indoles derivatives employing environmentally friendly wide range of basic catalyst ${ }^{19-21}$.

\section{RESULT AND DISCUSSION}

\section{1. Chemistry}

The synthesis of pyrano[2,3-b]indoles derivatives was performed as shown in Scheme 1. In the initial step, oxiindole [1] was synthesized by reaction of isatin with hydrazine hydrate in reflux temperature for $3 \mathrm{hr}$. The pyrano[2,3-b]indoles derivatives HB-401-410 were synthesized by the reaction of the oxiindole [1] with aromatic aldehyde and malononitrile/ethylcyano acetate in the presence of basic catalyst. The purity of the compounds was determined by TLC and elemental analyses.<smiles>[R2]CC#N</smiles><smiles>[R2]C1=C(N)Oc2[nH]c3ccccc3c2C1[R]</smiles>

\section{HB-401 to HB-410}

Scheme 1. Reagents and conditions: (a) ethanol, piperidine/pyrrolidine/pyridine, where: stirring, $30 \mathrm{~min}$;

$\mathrm{R}_{1}=$ various substituted phenyl group,

$\mathrm{R}_{2}=\mathrm{CN}$, COOEt

Table 1. Synthesis of substituted pyrano[2,3-b]indoles derivatives.

\begin{tabular}{ccccccc}
\hline Code & $\mathbf{R}_{\mathbf{1}}$ & $\mathbf{R}_{\mathbf{2}}$ & M.F. & M.W. & M.P. ${ }^{\circ} \mathbf{C}$ & $\mathbf{R}_{\mathbf{f}}$ \\
\hline $\mathrm{HB}-401$ & $4-\mathrm{CH}_{3} \mathrm{C}_{6} \mathrm{H}_{5}$ & $\mathrm{CN}$ & $\mathrm{C}_{19} \mathrm{H}_{15} \mathrm{~N}_{3} \mathrm{O}$ & 301 & $168-171$ & 0.55 \\
$\mathrm{HB}-402$ & $4-\mathrm{OCH}_{3} \mathrm{C}_{6} \mathrm{H}_{5}$ & $\mathrm{CN}$ & $\mathrm{C}_{19} \mathrm{H}_{15} \mathrm{~N}_{3} \mathrm{O}_{2}$ & 317 & $188-190$ & 0.51 \\
$\mathrm{HB}-403$ & $3,4-\mathrm{OCH}_{3} \mathrm{C}_{6} \mathrm{H}_{5}$ & $\mathrm{CN}$ & $\mathrm{C}_{20} \mathrm{H}_{17} \mathrm{~N}_{3} \mathrm{O}_{3}$ & 347 & $184-186$ & 0.61 \\
$\mathrm{HB}-404$ & $2,5-\mathrm{OCH}_{3} \mathrm{C}_{6} \mathrm{H}_{5}$ & $\mathrm{CN}$ & $\mathrm{C}_{20} \mathrm{H}_{17} \mathrm{~N}_{3} \mathrm{O}_{3}$ & 347 & $188-190$ & 0.57 \\
$\mathrm{HB}-405$ & $3,4,5-\mathrm{OCH}_{3} \mathrm{C}_{6} \mathrm{H}_{5}$ & $\mathrm{CN}$ & $\mathrm{C}_{21} \mathrm{H}_{19} \mathrm{~N}_{3} \mathrm{O}_{4}$ & 377 & $180-182$ & 0.48 \\
$\mathrm{HB}-406$ & $4-\mathrm{CH}_{3} \mathrm{C}_{6} \mathrm{H}_{5}$ & $\mathrm{COOEt}$ & $\mathrm{C}_{21} \mathrm{H}_{20} \mathrm{~N}_{2} \mathrm{O}_{3}$ & 348 & $181-183$ & 0.60 \\
\hline
\end{tabular}




\begin{tabular}{lcccccc}
\hline HB-407 & $4-\mathrm{OCH}_{3} \mathrm{C}_{6} \mathrm{H}_{5}$ & COOEt & $\mathrm{C}_{21} \mathrm{H}_{20} \mathrm{~N}_{2} \mathrm{O}_{4}$ & 364 & $188-190$ & 0.52 \\
HB-408 & $3,4-\mathrm{OCH}_{3} \mathrm{C}_{6} \mathrm{H}_{5}$ & COOEt & $\mathrm{C}_{22} \mathrm{H}_{22} \mathrm{~N}_{2} \mathrm{O}_{5}$ & 394 & $218-220$ & 0.62 \\
HB-409 & $2,5-\mathrm{OCH}_{3} \mathrm{C}_{6} \mathrm{H}_{5}$ & COOEt & $\mathrm{C}_{22} \mathrm{H}_{22} \mathrm{~N}_{2} \mathrm{O}_{5}$ & 394 & $148-150$ & 0.50 \\
HB-410 & $3,4,5-\mathrm{OCH}_{3} \mathrm{C}_{6} \mathrm{H}_{5}$ & COOEt & $\mathrm{C}_{23} \mathrm{H}_{24} \mathrm{~N}_{2} \mathrm{O}_{6}$ & 424 & $198-200$ & 0.56 \\
\hline
\end{tabular}

TLC Solvent system: Hexane: Ethyl acetate - 4:6.

Several methods are used in the synthesis of these pyrano[2,3-b]indoles derivatives. The synthesis of these heterocycles has been usually carried out in polar protic organic solvents such as water, methanol and polar aprotic organic solvents such as acetonitrile, DMF and DMSO. The choice of a solvent is a crucial factor for multicomponent reactions. So at the first step we looked into the solvent selection for this reaction. We had selected protic solvent ethanol for this reaction. Apart from the solvent, the efficiency of the multicomponent reactions is mainly affected by the catalyst and the reaction time. Here we employed variety of basic catalyst (piperidine/pyrrolidine/pyridine) and observed time for completion of reaction. Basicity of catalyst affect on the rate of reaction. More basic catalyst completed reaction fast than other. So, here when reaction is carried out using piperidine as catalyst reaction takes less time for completion, while for pyrrolidine and pyridine take more time for completion.

Table 2. Effects of different catalysts on the reaction productivity.

\begin{tabular}{ccccccc}
\hline \multirow{2}{*}{ Code } & \multicolumn{2}{c}{ Piperidine } & \multicolumn{2}{c}{ Pyrrolidine } & \multicolumn{2}{c}{ Pyridine } \\
\cline { 2 - 7 } & $\begin{array}{c}\text { Yield } \\
\text { \% }\end{array}$ & $\begin{array}{c}\text { Time } \\
\text { (minute) }\end{array}$ & $\begin{array}{c}\text { Yield } \\
\text { \% }\end{array}$ & $\begin{array}{c}\text { Time } \\
\text { (minute) }\end{array}$ & $\begin{array}{c}\text { Yield } \\
\text { \% }\end{array}$ & $\begin{array}{c}\text { Time } \\
\text { (minute) }\end{array}$ \\
\hline HB-401 & 89 & 15 & 82 & 25 & 74 & 30 \\
HB-402 & 87 & 15 & 82 & 25 & 71 & 30 \\
HB-403 & 89 & 15 & 80 & 25 & 77 & 30 \\
HB-404 & 90 & 15 & 84 & 25 & 75 & 30 \\
HB-405 & 84 & 15 & 81 & 25 & 72 & 30 \\
HB-406 & 85 & 15 & 81 & 25 & 74 & 30 \\
HB-407 & 88 & 15 & 82 & 25 & 72 & 30 \\
HB-408 & 87 & 15 & 82 & 25 & 71 & 30 \\
HB-409 & 86 & 15 & 80 & 25 & 74 & 30 \\
HB-410 & 85 & 15 & 78 & 25 & 73 & 30 \\
\hline
\end{tabular}


<smiles>O=Cc1ccccc1</smiles><smiles>[R]CNCC</smiles>

Base<smiles>[R]C=C(C=CC=C)C=C([R2])C#N</smiles><smiles>O=C1Cc2ccccc2N1</smiles>

Base<smiles>[R][X]c1ccccc1C(C([R])C#N)C1C(=O)Nc2ccccc21</smiles>

1<smiles>[R]CC(c1ccccc1)C(c1ccccc1)C([R])C#N</smiles><smiles>[CH]=C</smiles>

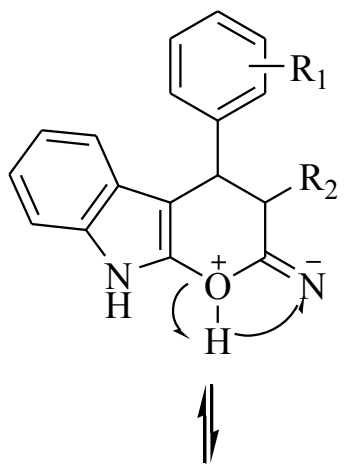<smiles>CCC</smiles><smiles>[R]c1cccc(C(c2c(O)[nH]c3ccccc23)C(CC#N)C([R])CC)c1</smiles><smiles></smiles><smiles>[R]c1ccccc1C1c2c([nH]c3ccccc23)OC(=N)C1[R2]</smiles><smiles>[R][X]c1ccccc1C1C([R])=C(N)Oc2[nH]c3ccccc3c21</smiles>

Scheme 2. Plausible mechanism for the formation of substituted pyrano[2,3-b]indoles using base as catalyst.

Possible mechanism for the base catalyzed synthesis of substituted pyrano[2,3-b]indoles has been proposed in Scheme 2. In summary, this paper describes a convenient and efficient process for the synthesis of substituted pyrano[2,3-b]indoles through the three components coupling of aldehydes, pyrazolone and malononitrile using variety of base as a catalyst. Reaction profile is very clean and no side products are formed. All the synthesized pyrano[2,3b]indoles have been characterized on the basis of elemental and spectral studies.

\section{EXPERIMENTAL SECTION}

All research chemicals were purchased from Sigma-Aldrich and used as such for the reactions. Reactions were monitored by thin-layer chromatography (TLC) on pre-coated silica gel GF254 plates from E-Merck Co and compounds visualized either by exposure to UV light or staining with reagents. Melting points were determined in open capillaries and are uncorrected. The IR spectra were recorded on SHIMADZU-FTIR-8400 spectrophotometer using $\mathrm{KBr}$ pellet method. ${ }^{1} \mathrm{H}$ NMR spectra were recorded on Bruker 300$\mathrm{MHz}$ NMR spectrometer in $\mathrm{CDCl}_{3}$ with TMS as internal standard. Mass spectrum was 
recorded on JOEL SX 102/DA-600-Mass spectrometer and elemental analysis was carried out using Heraus $\mathrm{C}, \mathrm{H}$, and $\mathrm{N}$ rapid analyser.

\section{1. General procedure for the synthesis of 2-amino-4,9-dihydro-4-phenylpyrano[2,3-b] indole-3-carbonitriles/ethyl-carboxylates (HB-401-410)}

At first, substituted benzaldehyde $(0.01 \mathrm{~mol})$, malononitrile/ethylcyano acetate $(0.01$ mol) and piperidine/pyridine/pyrrolidine as a catalyst (1 drop) and ethanol as solvent was stirred for $2 \mathrm{~min}$, then oxiindole $(0.01 \mathrm{~mol})$ was added to this mixture and stirred at room temperature for appropriate time. The reaction was monitored by TLC. The solid was falls out which indicates completion of reaction. Solids was filtered out and washed by cold ethanol and after that was crystallized from hot ethanol to afford the pure products. Some derivatives have good crystalline property while most of were fluffy in nature.

\section{2-amino-4,9-dihydro-4-p-tolylpyrano[2,3-b]indole-3-carbonitrile (HB-401)}

Mp 168-171 ${ }^{\circ} \mathrm{C}$; IR $\left(\mathrm{cm}^{-1}\right): 3473(\mathrm{~N}-\mathrm{H}$ stretching of free primary amine), $3227(\mathrm{~N}-\mathrm{H}$ stretching of pyrrole ring), 3117 (C-H stretching of aromatic ring), 2196 ( $\mathrm{C} \equiv \mathrm{N}$ stretching of the nitrile group), 1600 (N-H deformation pyrrole ring), 1053 (C-H in plane bending of aromatic ring), 806 (C-H out of plane bending for 1,4-disubstituted aromatic ring); $1 \mathrm{H}$ NMR(DMSO- $d 6,400 \mathrm{MHz}): \delta(\mathrm{ppm}) 2.18-2.24(\mathrm{t}, \quad J=1.1 \mathrm{~Hz}, 3 \mathrm{H}), 5.1-5.17(\mathrm{~d}, J=1.0 \mathrm{~Hz}$, $1 \mathrm{H}), 6.78-6.87(\mathrm{td}, J=7.5,1.5 \mathrm{~Hz}, 1 \mathrm{H}), 6.97-7.07(\mathrm{td}, J=7.5,1.5 \mathrm{~Hz}, 1 \mathrm{H}), 7.08-7.17(\mathrm{dq}, J=$ 7.3, 1.1 Hz, 2H), 7.51-7.59 (m, 2H), 7.60-7.67 (dd, $J=7.5,1.5 \mathrm{~Hz}, 1 \mathrm{H}), 7.74-7.82$ (dd, $J=$ 7.5, 1.6 Hz, 1H) MS: m/z 301; Anal. Calcd. for $\mathrm{C}_{19} \mathrm{H}_{15} \mathrm{~N}_{3} \mathrm{O}: \mathrm{C}, 75.73 ; \mathrm{H}, 5.02 ; \mathrm{N}, 13.94$. Found: C, 75.71; H, 5.04; N, $13.95 \%$.

\section{2-amino-4,9-dihydro-4-(4-methoxyphenyl)pyrano[2,3-b]indole-3-carbonitrile (HB-402)}

Mp 188-190 ${ }^{\circ} \mathrm{C}$; IR $\left(\mathrm{cm}^{-1}\right): 3470(\mathrm{~N}-\mathrm{H}$ stretching of free primary amine), $3220(\mathrm{~N}-\mathrm{H}$ stretching of pyrrole ring), 3101 (C-H stretching of aromatic ring), $2190(\mathrm{C} \equiv \mathrm{N}$ stretching of the nitrile group), 1624 ( $\mathrm{N}-\mathrm{H}$ deformation pyrrole ring), 1050 (C-H in plane bending of aromatic ring), 807 (C-H out of plane bending for 1,4-disubstituted aromatic ring); $1 \mathrm{H}$ NMR(DMSO-d6, $400 \mathrm{MHz}): \delta(\mathrm{ppm}) 3.76-3.81(\mathrm{~s}, 3 \mathrm{H}), 5.09-5.14(\mathrm{t}, J=0.9 \mathrm{~Hz}, 1 \mathrm{H}), 6.78-$ $6.89(\mathrm{~m}, 3 \mathrm{H}), 6.97-7.07(\mathrm{td}, J=7.5,1.5 \mathrm{~Hz}, 1 \mathrm{H}), 7.30-7.38(\mathrm{~m}, 2 \mathrm{H}), 7.60-7.67$ (dd, $J=7.4$, $1.5 \mathrm{~Hz}, 1 \mathrm{H}), 7.73-7.81(\mathrm{dd}, J=7.4,1.6 \mathrm{~Hz}, 1 \mathrm{H})$; MS: $m / z$ 317; Anal. Calcd. for $\mathrm{C}_{19} \mathrm{H}_{15} \mathrm{~N}_{3} \mathrm{O}_{2}$ : C, 71.91; H, 4.76; N, 13.24. Found: C, 71.90; H, 4.74; N, $13.23 \%$.

2-amino-4,9-dihydro-4-(3,4-dimethoxyphenyl)pyrano[2,3-b]indole-3-carbonitrile (HB403)

Mp 184-186 ${ }^{\circ} \mathrm{C}$; IR $\left(\mathrm{cm}^{-1}\right): 3460(\mathrm{~N}-\mathrm{H}$ stretching of free primary amine), $3241(\mathrm{~N}-\mathrm{H}$ stretching of pyrrrol ring), 3121 (C-H stretching of aromatic ring), 2181 ( $\mathrm{C} \equiv \mathrm{N}$ stretching of the nitrile group), $1620(\mathrm{~N}-\mathrm{H}$ deformation pyrrole ring), $1050(\mathrm{C}-\mathrm{H}$ in plane bending of aromatic ring), 820 (C-H out of plane bending for 1,4-disubstituted aromatic ring); $1 \mathrm{H}$ NMR(DMSO- $d 6,400 \mathrm{MHz}): \delta$ (ppm) 3.72-3.77 (s, 3H), 3.78-3.83 (s, 3H), 5.10-5.15 (t, $J=$ $1.0 \mathrm{~Hz}, 1 \mathrm{H}), 6.78-6.87(\mathrm{td}, J=7.5,1.5 \mathrm{~Hz}, 1 \mathrm{H}), 6.97-7.07(\mathrm{td}, J=7.5,1.5 \mathrm{~Hz}, 1 \mathrm{H}), 7.11-7.20$ $(\mathrm{m}, 2 \mathrm{H}), 7.24-7.32$ (ddd, $J=7.5,2.0,1.0 \mathrm{~Hz}, 1 \mathrm{H}), 7.60-7.68(\mathrm{dd}, J=7.5,1.5 \mathrm{~Hz}, 1 \mathrm{H}), 7.73-$ $7.81(\mathrm{dd}, J=7.5,1.5 \mathrm{~Hz}, 1 \mathrm{H})$; MS: $m / z$ 347; Anal. Calcd. for $\mathrm{C}_{20} \mathrm{H}_{17} \mathrm{~N}_{3} \mathrm{O}_{3}$ : C, 69.15; $\mathrm{H}, 4.93$; N, 12.10. Found: C, 69.17; H, 4.90; N, $12.12 \%$.

2-amino-4,9-dihydro-4-(2,5-dimethoxyphenyl)pyrano[2,3-b]indole-3-carbonitrile (HB404)

Mp 188-190 ${ }^{\circ} \mathrm{C}$; IR $\left(\mathrm{cm}^{-1}\right)$ : $3454(\mathrm{~N}-\mathrm{H}$ stretching of free primary amine), $3221(\mathrm{~N}-\mathrm{H}$ stretching of pyrrole ring), $3110(\mathrm{C}-\mathrm{H}$ stretching of aromatic ring), $2188(\mathrm{C} \equiv \mathrm{N}$ stretching of 
the nitrile group), $1622(\mathrm{~N}-\mathrm{H}$ deformation pyrrole ring), $1050(\mathrm{C}-\mathrm{H}$ in plane bending of aromatic ring), 812 (C-H out of plane bending for 1,4-disubstituted aromatic ring); $1 \mathrm{H}$ NMR(DMSO- $d 6,400 \mathrm{MHz}): \delta(\mathrm{ppm}) 3.69-4.15(\mathrm{~d}, J=8.1 \mathrm{~Hz}, 6 \mathrm{H}), 5.07-5.16(\mathrm{~d}, J=1.1 \mathrm{~Hz}$, $1 \mathrm{H}), 6.78-7.16(\mathrm{~m}, 3 \mathrm{H}), 7.23-7.37(\mathrm{dd}, J=2.0,1.0 \mathrm{~Hz}, 1 \mathrm{H}), 7.60-7.71(\mathrm{~m}, 1 \mathrm{H}), 7.75-8.00$ (dd, $J=7.5,1.6 \mathrm{~Hz}, 1 \mathrm{H})^{;} \mathrm{MS}: m / z$ 347; Anal. Calcd. for $\mathrm{C}_{20} \mathrm{H}_{17} \mathrm{~N}_{3} \mathrm{O}_{3}: \mathrm{C}, 69.15 ; \mathrm{H}, 4.93$; N, 12.10. Found: C, 69.16; H, 4.90; N, $12.10 \%$.

\section{2-amino-4,9-dihydro-4-(3,4,5-trimethoxyphenyl)pyrano[2,3-b]indole-3-carbonitrile (HB-405)}

Mp 180-182 ${ }^{\circ} \mathrm{C}$; IR $\left(\mathrm{cm}^{-1}\right): 3470(\mathrm{~N}-\mathrm{H}$ stretching of free primary amine), $3231(\mathrm{~N}-\mathrm{H}$ stretching of pyrrole ring), 3117 (C-H stretching of aromatic ring), $2196(\mathrm{C} \equiv \mathrm{N}$ stretching of the nitrile group), 1600 ( $\mathrm{N}-\mathrm{H}$ deformation pyrazole ring), $1063(\mathrm{C}-\mathrm{H}$ in plane bending of aromatic ring), 810 (C-H out of plane bending for 1,4-disubstituted aromatic ring); $1 \mathrm{H}$ NMR(DMSO- $d 6,400 \mathrm{MHz}): \delta(\mathrm{ppm}) 3.66-3.71(\mathrm{~s}, 9 \mathrm{H}), 5.12-5.17(\mathrm{~m}, 1 \mathrm{H}), 6.78-6.87(\mathrm{td}, J=$ $7.5,1.5 \mathrm{~Hz}, 1 \mathrm{H}), 6.91-6.96(\mathrm{~d}, J=1.1 \mathrm{~Hz}, 2 \mathrm{H}), 6.97-7.07(\mathrm{td}, J=7.5,1.5 \mathrm{~Hz}, 1 \mathrm{H}), 7.60-7.67$ (dd, $J=7.5,1.6 \mathrm{~Hz}, 1 \mathrm{H}), 7.76-7.83(\mathrm{dd}, J=7.5,1.5 \mathrm{~Hz}, 1 \mathrm{H})$; MS: $m / z$ 377; Anal. Calcd. for $\mathrm{C}_{21} \mathrm{H}_{19} \mathrm{~N}_{3} \mathrm{O}_{4}$ : C, 66.83; H, 5.07; N, 11.13. Found: C, 66.80; H, 5.04; N, $11.16 \%$.

Ethyl-2-amino-4,9-dihydro-4-p-tolylpyrano[2,3-b]indole-3-carboxylate (HB-406)

Mp 181-183 ${ }^{\circ} \mathrm{C}$; IR $\left(\mathrm{cm}^{-1}\right): 3470(\mathrm{~N}-\mathrm{H}$ stretching of free primary amine), $3220(\mathrm{~N}-\mathrm{H}$ stretching of pyrrole ring), 3110 (C-H stretching of aromatic ring), $2190(\mathrm{C} \equiv \mathrm{N}$ stretching of the nitrile group), 1622 (N-H deformation pyrrole ring), $1041(\mathrm{C}-\mathrm{H}$ in plane bending of aromatic ring), 813 (C-H out of plane bending for 1,4-disubstituted aromatic ring); $1 \mathrm{H}$ NMR(DMSO- $d 6,400 \mathrm{MHz}): \delta(\mathrm{ppm}) 1.09-1.17(\mathrm{t}, J=5.9 \mathrm{~Hz}, 3 \mathrm{H}), 2.18-2.24(\mathrm{t}, J=1.1 \mathrm{~Hz}$, $3 \mathrm{H}), 4.00-4.10(\mathrm{q}, J=5.9 \mathrm{~Hz}, 2 \mathrm{H}), 5.48-5.54(\mathrm{~d}, J=1.1 \mathrm{~Hz}, 1 \mathrm{H}), 6.78-6.87(\mathrm{td}, J=7.5,1.5$ $\mathrm{Hz}, 1 \mathrm{H}), 6.97-7.13(\mathrm{~m}, 3 \mathrm{H}), 7.49-7.57(\mathrm{~m}, 2 \mathrm{H}), 7.60-7.67(\mathrm{dd}, J=7.4,1.6 \mathrm{~Hz}, 1 \mathrm{H}), 7.75-7.82$ (dd, $J=7.4,1.6 \mathrm{~Hz}, 1 \mathrm{H})$; MS: $m / z$ 348; Anal. Calcd. for $\mathrm{C}_{21} \mathrm{H}_{20} \mathrm{~N}_{2} \mathrm{O}_{3}: \mathrm{C}, 72.40 ; \mathrm{H}, 5.79 ; \mathrm{N}$, 8.04. Found: C, 72.42; H, 5.77; N, $8.06 \%$.

\section{Ethyl-2-amino-4,9-dihydro-4-(4-methoxyphenyl)pyrano[2,3-b]indole-3-carboxylate (HB-407)}

Mp 188-190 ${ }^{\circ} \mathrm{C}$; IR $\left(\mathrm{cm}^{-1}\right)$ : $3431(\mathrm{~N}-\mathrm{H}$ stretching of free primary amine), $3200(\mathrm{~N}-\mathrm{H}$ stretching of pyrrole ring), 3127 (C-H stretching of aromatic ring), $2210(\mathrm{C} \equiv \mathrm{N}$ stretching of the nitrile group), 1618 ( $\mathrm{N}-\mathrm{H}$ deformation pyrrole ring), $1050(\mathrm{C}-\mathrm{H}$ in plane bending of aromatic ring), 810 (C-H out of plane bending for 1,4-disubstituted aromatic ring); $1 \mathrm{H}$ NMR(DMSO- $d 6,400 \mathrm{MHz}): \delta(\mathrm{ppm}) 1.09-1.17(\mathrm{t}, J=5.9 \mathrm{~Hz}, 3 \mathrm{H}), 3.76-3.81(\mathrm{~s}, 3 \mathrm{H}), 4.00-$ $4.10(\mathrm{q}, J=5.9 \mathrm{~Hz}, 2 \mathrm{H}), 5.48-5.53(\mathrm{t}, J=1.0 \mathrm{~Hz}, 1 \mathrm{H}), 6.77-6.87(\mathrm{~m}, 3 \mathrm{H}), 6.97-7.07(\mathrm{td}, J=$ 7.5, $1.5 \mathrm{~Hz}, 1 \mathrm{H}), 7.27-7.35(\mathrm{~m}, 2 \mathrm{H}), 7.60-7.67(\mathrm{dd}, J=7.4,1.5 \mathrm{~Hz}, 1 \mathrm{H}), 7.75-7.82(\mathrm{dd}, J=$ 7.4, 1.6 Hz, 1H); MS: $m / z$ 364; Anal. Calcd. for $\mathrm{C}_{21} \mathrm{H}_{20} \mathrm{~N}_{2} \mathrm{O}_{4}$ : C, 69.22; $\mathrm{H}, 5.53 ; \mathrm{N}, 7.69$. Found: C, 69.20; H, 5.50; N, 7.71\%.

\section{Ethyl-2-amino-4,9-dihydro-4-(3,4-dimethoxyphenyl)pyrano[2,3-b]indole-3-carboxylate (HB-408)}

Mp 218-220 ${ }^{\circ} \mathrm{C}$; IR $\left(\mathrm{cm}^{-1}\right)$ : $3466(\mathrm{~N}-\mathrm{H}$ stretching of free primary amine), $3225(\mathrm{~N}-\mathrm{H}$ stretching of pyrrole ring), 3110 (C-H stretching of aromatic ring), $2188(\mathrm{C} \equiv \mathrm{N}$ stretching of the nitrile group), 1578 (N-H deformation pyrrole ring), $1050(\mathrm{C}-\mathrm{H}$ in plane bending of aromatic ring), 818 (C-H out of plane bending for 1,4-disubstituted aromatic ring); $1 \mathrm{H}$ NMR(DMSO- $d 6,400 \mathrm{MHz}): \delta(\mathrm{ppm}) 1.09-1.17(\mathrm{t}, J=5.9 \mathrm{~Hz}, 3 \mathrm{H}), 3.72-3.77(\mathrm{~s}, 3 \mathrm{H}), 3.78-$ $3.83(\mathrm{~s}, 3 \mathrm{H}), 4.00-4.10(\mathrm{q}, J=5.9 \mathrm{~Hz}, 2 \mathrm{H}), 5.49-5.54(\mathrm{t}, J=0.9 \mathrm{~Hz}, 1 \mathrm{H}), 6.78-6.87(\mathrm{td}, J=$ 7.5, $1.5 \mathrm{~Hz}, 1 \mathrm{H}), 6.97-7.15(\mathrm{~m}, 3 \mathrm{H}), 7.21-7.29$ (ddd, $J=7.7,2.0,1.0 \mathrm{~Hz}, 1 \mathrm{H}), 7.60-7.67$ (dd, 
$J=7.5,1.5 \mathrm{~Hz}, 1 \mathrm{H}), 7.76-7.84(\mathrm{dd}, J=7.5,1.6 \mathrm{~Hz}, 1 \mathrm{H}) \mathrm{MS}: m / z$ 394; Anal. Calcd. for $\mathrm{C}_{22} \mathrm{H}_{22} \mathrm{~N}_{2} \mathrm{O}_{5}$ : C, 66.99; H, 5.62; N, 7.10. Found: C, 66.94; H, 5.60; N, 7.14 \%.

\section{Ethyl-2-amino-4,9-dihydro-4-(2,5-dimethoxyphenyl)pyrano[2,3-b]indole-3-carboxylate (HB-409)}

Mp 148-150 ${ }^{\circ} \mathrm{C}$; IR $\left(\mathrm{cm}^{-1}\right): 3477(\mathrm{~N}-\mathrm{H}$ stretching of free primary amine), $3243(\mathrm{~N}-\mathrm{H}$ stretching of pyrrole ring), 3128 (C-H stretching of aromatic ring), 2181 (C $\equiv \mathrm{N}$ stretching of the nitrile group), 1617 ( $\mathrm{N}-\mathrm{H}$ deformation pyrrole ring), 1054 (C-H in plane bending of aromatic ring), $836(\mathrm{C}-\mathrm{H}$ out of plane bending for 1,4-disubstituted aromatic ring); $1 \mathrm{H}$ NMR(DMSO- $d 6,400 \mathrm{MHz}): \delta(\mathrm{ppm}) 1.09-1.17(\mathrm{t}, J=5.9 \mathrm{~Hz}, 3 \mathrm{H}), 3.69-3.77(\mathrm{~d}, J=8.1 \mathrm{~Hz}$, $6 \mathrm{H}), 4.00-4.10(\mathrm{q}, J=5.9 \mathrm{~Hz}, 2 \mathrm{H}), 5.73-5.78(\mathrm{~d}, J=1.1 \mathrm{~Hz}, 1 \mathrm{H}), 6.78-6.87(\mathrm{~m}, 2 \mathrm{H}), 6.95-$ $7.07(\mathrm{~m}, 3 \mathrm{H}), 7.60-7.67(\mathrm{dd}, J=7.5,1.6 \mathrm{~Hz}, 1 \mathrm{H}), 7.79-7.86(\mathrm{dd}, J=7.5,1.6 \mathrm{~Hz}, 1 \mathrm{H})$; MS: $m / z$ 394; Anal. Calcd. for $\mathrm{C}_{22} \mathrm{H}_{22} \mathrm{~N}_{2} \mathrm{O}_{5}$ : C, 66.99; H, 5.62; N, 7.10. Found: C, 66.99; H, 5.62; $\mathrm{N}, 7.10 \%$.

\section{Ethyl-2-amino-4,9-dihydro-4-(3,4,5-trimethoxyphenyl)pyrano[2,3-b]indole-3- carboxylate (HB-410)}

Mp 198-200 ${ }^{\circ} \mathrm{C}$; IR $\left(\mathrm{cm}^{-1}\right): 3470(\mathrm{~N}-\mathrm{H}$ stretching of free primary amine), $3231(\mathrm{~N}-\mathrm{H}$ stretching of pyrrole ring), 3111 (C-H stretching of aromatic ring), 2191 ( $\mathrm{C} \equiv \mathrm{N}$ stretching of the nitrile group), $1612(\mathrm{~N}-\mathrm{H}$ deformation pyrrole ring), 1042 (C-H in plane bending of aromatic ring), 800 (C-H out of plane bending for 1,4-disubstituted aromatic ring); $1 \mathrm{H}$ NMR(DMSO- $d 6,400 \mathrm{MHz}$ ): $\delta(\mathrm{ppm}) 1.09-1.17$ (t, $J=5.9 \mathrm{~Hz}, 3 \mathrm{H}), 3.66-3.71$ (s, 9H), $4.00-$ $4.10(\mathrm{q}, J=5.9 \mathrm{~Hz}, 2 \mathrm{H}), 5.55-5.57(\mathrm{~m}, 1 \mathrm{H}), 6.78-6.87(\mathrm{td}, J=7.5,1.5 \mathrm{~Hz}, 1 \mathrm{H}), 6.88-6.94(\mathrm{~d}$, $J=1.1 \mathrm{~Hz}, 2 \mathrm{H}), 6.97-7.07$ (td, $J=7.5,1.5 \mathrm{~Hz}, 1 \mathrm{H}), 7.60-7.67$ (dd, $J=7.5,1.5 \mathrm{~Hz}, 1 \mathrm{H}), 7.76-$ 7.83 (dd, $J=7.5,1.6 \mathrm{~Hz}, 1 \mathrm{H}$ ); MS: $m / z$ 424; Anal. Calcd. for $\mathrm{C}_{22} \mathrm{H}_{24} \mathrm{~N}_{2} \mathrm{O}_{6}$ : C, 65.08; $\mathrm{H}, 5.70$; N, 6.60. Found: C, 65.11; H, 5.67; N, $6.62 \%$.

\section{CONCLUSION}

In conclusion, we have developed a highly efficient base catalyzed, one pot, three component protocol for the synthesis of pyrano[2,3-b]indoles via condensation of 1,3bifunctional synthon which is oxiindole, aromatic aldehyde and malononitrile/ethylcyano acetate in the presence of base catalyst. The advantages of this method are clean reaction, short reaction time, high yield, easy purification and economic availability of the catalyst.

\section{ACKNOWLEDGEMENT}

Authors are thankful to the Professor and Head, Department of Chemistry, Saurashtra University-Rajkot for providing necessary laboratory facilities.

\section{References}

[1] D. M. D'Souza, T. J. Müller, Chem. Soc. Rev. 36 (2007) 1095.

[2] A. Domling, Chem. Rev. 106 (2006) 17.

[3] I. Ugi, Pure and Appl. Chem. 73 (2001) 187.

[4] F. Lie'by-Muller, C. Simon, T. Constantieux, J. Rodriguez, Comb. Sci. 25 (2006 ) 432. 
[5] C. Simon, T. Constantieux, J. Rodriguez, Eur. J. Org. Chem. 24 (2004) 4957.

[6] N. Evdokimov, A. Kireev, A. Yakovenko, M. Antipin, I. Magedov, J. Kornienko, J. Org. Chem. 72 (2007) 3443.

[7] J. L. Wang, D. Liu, Z. J. Zhang, S. Shan, X. Han, S. M. Srinivasula, C. M. Croce, E. S. Alnemri, Z. Huang, Proc. Natl. Acad. Sci. U.S.A. 97 (2000) 7124.

[8] E. S. El-Tamany, F. A. El-Shahed, B. H. Mohamed, J. Serb. Chem. Soc. 64 (1999) 9.

[9] M. E. A. Zaki, H. A. Soliman, O. A. Hiekal, A. E. Rashad, Z. Naturforsch. C. 61c (2006) 1 .

[10] F. M. Abdelrazek, P. Metz, N. H. Metwally, S. F. El-Mahrouky, Arch. Pharm. 339 (2006) 456.

[11] F. M. Abdelrazek, P. Metz, O. Kataeva, A. Jager, S. F. EI-Mahrouky, Arch. Pharm. 340 (2007) 543

[12] N. Foloppe, L. M. Fisher, R. Howes, A. Potter, A. G. S. Robertson, A. E. Surgenor, Bioorg. Med. Chem. 14 (2006) 4792.

[13] V. Y. Sosnovskikh, M. A. Barabanov, B. I. Usachev, R. A. Irgashev, V. S. Moshkin, Russ. Chem. Bull., Int. Ed. 54 (2005) 2846.

[14] H. Junek, H. Aigner, Chem. Ber. 106 (1973) 914.

[15] H. H. Otto, Arch. Pharm. 307 (1974) 444.

[16] Yu. A. Sharanin, L. G. Sharanina, V. V. Puzanova, Zh. Org. Khim. 19 (1983) 2609.

[17] K. Kuppusamy, P. Kasi, Tetrahedron Lett. 51 (2010) 3312.

[18] H. Mecadon, M. R. Rohman, M. Rajbangshi, B. Myrboh, Tetrahedron Lett. 52 (2011) 2523.

[19] J. W. Pavlik, V. Ervithayasuporn, J. C. MacDonald, S. Tantayanon, ARKIVOC 8 (2009) 57.

[20] Ratnamala P. Sonawane, Rahul R. Tripathi, International Letters of Chemistry, Physics and Astronomy 7(1) (2013) 30-36.

[21] Rahul R. Tripathi, Ratnamala P. Sonawane, International Letters of Chemistry, Physics and Astronomy 10(2) (2013) 119-125. 